\title{
Material Atmospheres:
}

\section{Theorising Recent Shifts in Interior Visualisation}

Gregory Marinic

University of Kentucky

USA

\begin{abstract}
Much like Walter Benjamin's analysis of the Parisian arcades during the interwar years of the early 20th century, emerging methods of seeing interior spaces reveal a deeper gaze into the contextual, material, and phenomenological conditions that produce more nuanced visions of interiority. A collective consciousness surrounding these constructed narratives is reflected in charged associations with the most salient imperatives of our time-globalisation, resource depletion, ecological degradation, and political instability-as well as their corresponding effects on the built environment. These visual provocations have incrementally percolated up to embody an expanding field of design activism for educators, theorists, practitioners, and students. How do these avant-garde techniques operate? What do they reveal about socio-political, economic, and consumptive forces shaping the global built environment? How do these speculative methods offer more critical ways to communicate dynamic conditions?
\end{abstract}

Keywords: visualisation, interiors, architecture, aura, materiality, theory, Walter Benjamin, Gilles Deleuze, Félix Guattari 
The arcades and interiors, the exhibition halls and panoramas are residues of a dream world. The utilisation of dream elements when waking up is a textbook example of dialectical thought. Hence, dialectical thought is the organ of historical awakening. After all, each epoch not only dreams the next, but also in dreaming, strives toward awakening.

Walter Benjamin, The Arcades Project

In his fragmented and unfinished The Arcades Project, Walter Benjamin studied the interior conditions of the Parisian arcades to document and analyse simultaneous forces shaping the broader city. Confronting the arcades during the interwar years of the early twentieth century, Benjamin assessed how their ageing surfaces and contents contrasted with the rapid rise of modernism. He reflected on the recent past to examine the transient subcultures that flourished in these forgotten spaces. Benjamin used dialectical, or comparative, images as his primary analytical device. Placing particular emphasis on how the arcades had been rejected by modernity, he studied their shifting influence on consumption, social organisation, and urbanity.

In recent years, architectural visualisation techniques have developed a similar form of awareness vis-à-vis transience and change. Much like Benjamin, emerging methods of seeing interior spaces reveal a deeper gaze into the contextual, material, and phenomenological conditions that produce more nuanced visions of interiority. The collective consciousness surrounding these constructed narratives is reflected in charged associations with the most salient imperatives of our time-globalisation, resource depletion, ecological degradation, and political instability-as well as their corresponding effects on the built environment. These visual provocations have incrementally percolated up to embody an expanding field of design activism-by means of an alternative representational practice-for educators, theorists, practitioners, and students. How do these avant-garde techniques operate? What do they reveal about socio-political, economic, and consumptive forces shaping the global built environment? How do these speculative methods offer more critical ways to communicate dynamic conditions?

This article examines recent work in interior visualisation to demonstrate how forces of adaptation and inhabitation can be more proactively communicated. It mobilises theory to critique the ways in which emerging visual representational practices increasingly acknowledge unique spatial dynamics and material qualities. It begins by surveying the theoretical postulations of Walter Benjamin, 
as well as the post-structuralist ideas of Gilles Deleuze and Félix Guattari. Applying their perspectives to interpret recent shifts in visualisation culture, this study collects exemplars achieving greater complexity and sensorial qualities. In short, my research proposes a spatial lexicon inspired by the ponderings of Benjamin, Deleuze, and Guattari to taxonomise recent innovations expressing processes of time, appropriation, and transience.

\section{Walter Benjamin and Atmosphere}

In The Arcades Project, Walter Benjamin analysed the historical, social, and cultural influence of the nineteenth-century arcades in pre-World War II Paris to critique intersectional complexities of consumption and urban life (Ross, 2016). Benjamin encountered the Parisian arcades in the mid-1920s when they had already fallen into disrepair and partial abandonment. Unlike the Beaux-Arts train stations, bridges, and museums of the era, the faded arcades lacked formal monumentality and civic gravitas. Yet their diminutive scale and unique interior spatial qualities fostered rich social narratives to develop. As a building type that illustrated influences on industrialised society, the arcades fueled nineteenth-century consumption and the collective imagination (Horton \& Kraftl, 2014).

By the early twentieth century, the arcades had fallen out of fashion with the bourgeoisie and had transformed into places that sheltered marginalised communities from the larger city. These spaces incrementally developed into inclusive territories for diverse subcultures-artists, freethinkers, prostitutes, communists, and gays - that thrived in their seclusion. This hidden world of interior urban spaces spawned an avant-garde culture that Benjamin analysed and deconstructed (Chisholm, 2005). Furthermore, as a socio-geographic construct, the arcades embodied actions that rejected modernism and marked a transition away from consumption and toward a culture of growing alienation. Thus, Benjamin asserted that the Parisian arcade represented the most important building typology of the previous century, as well as a complex spatial environment that nurtured profound social multiplicity (Shields, 1994).

The temporal aspects of the arcades-the simultaneity of past traces, present conditions, and future dimensions-heightened Benjamin's desire to examine occupancies. And while he empathised with the zeitgeist of modernism, he did not suppress a long-held interest in the alienation of the flâneur, as well as the objects, social configurations, material conditions, and architectural 
artefacts of the past. His investigation of the arcades may be viewed as a unique form of spatial awareness shaped by layered theoretical influences. Benjamin theorised that the arcades existed in the present, yet negotiated manifold temporal domains. He applied the social theories of Freud and Marx, while considering Brecht's view that popular consumption could support the building of socialist utopias (Lunn, 1984). Furthermore, Benjamin believed that the analysis of language offered a portal into documenting spatial experience. The ideas of Nietzche, however, became a central theme in The Arcades Project which asserts that traces of the past latently shape the aesthetics of the present.

\section{Deleuze, Guattari, and the Plateaus}

In their book, A Thousand Plateaus: Capitalism and Schizophrenia, Deleuze and Guattari (1980) propose a postmodern social and cultural theory with strong connections to the human body. Their philosophical position-embodied by the concept of plateausblurs boundaries between reality, speculation, and authorship in the built environment. Thinking in terms of plateaus rather than beginnings and ends, they state:

There is no longer a tripartite between a field of reality (the world) and a field of representation (the book) and a field of subjectivity (the author), an assemblage establishes connections between certain multiplicities drawn from each of these orders. (Deleuze \& Guattari, 1980)

Their theory contributed significantly to the spatial cannon of Continental philosophy and may be applied anew to current methods of visualisation. The plateaus that Deleuze and Guattari identified describe anthropological, ontological, epistemological, political, and spatial implications that can be employed to articulate aspects of material culture. More specifically, I propose that the plateaus can be used as theoretical filters to identify emerging representational practices in architecture and interiors.

Extending these tripartite influences to visual culture-reality, representation, and subjectivity-addresses intersectional conditions that not only shape space, but also engage aspects of time and inhabitation. Thus, the plateaus may be reframed as taxonomic distinctions. On the one hand, visual representation methods translate ideas through sketches, drawings, imagery, and physical models to convey an unbuilt concept. As a form of design exploration, they allow projects to emerge through critical thinking and production. On the other, representational methods and the 
experiments that they explore uncover discursive elements through visual and haptic critique. In short, these techniques reconfigure spaces that already exist-without erasing literal and implied past traces-through speculative concepts embodied by the plateaus.

\section{Something Old, Something New}

Throughout time, unbuilt projects have exerted significant influence on visual representation ranging in scale from urbanism to interiors. Le Corbusier's utopian Ville Radieuse (1924) proposed a blueprint for social reform that radically transformed the design of twentieth-century cities worldwide. Mies van der Rohe's austere concept for the Friedrichstrasse Skyscraper Competition (1921) shifted our perceptions and expectations of the modern commercial workplace. Louis Boullée's monolithic Cenotaph for Newton (1784) expressed a sublime internal grandeur that continues to beguile contemporary designers. Today, continual shifts in design theory and production shape new visualisation practices through multidisciplinary work that has become more experimental and correspondingly less classifiable. Reflecting forces of globalisation and crisis, architects and designers are negotiating blurred disciplinary territories with alternative methodologies. As such, speculative representation techniques embody a disruptive discourse in projects that envision alternative futures for existing buildings and infrastructures.

At an interior scale, visualisations often describe spaces that have evolved over time, and thus, such drawings challenge conventional expectations of hegemony and sole-authorship. Unlike tabula rasa conditions, interior spaces often reveal a contributory blend of fixed and temporal elements. As a theoretical perspective demonstrating similarity, Benjamin's research on the Parisian arcades focused on the histories, occupancies, and atmospheres of inherited spaces. His example provides a theoretical foundation to reconsider trends operating within the contemporary interior representation.

For Benjamin, space represented the primary characteristic of Parisian arcades-a building type defined primarily by interiority with conventional exterior façades that were considered at once tenuous and secondary. He spoke of their constellation-like qualities evoking past traces that contrasted with conditions in the present. He hypothesised that interior space and temporality, rather than the external envelope and formality, represented the most salient characteristics of the arcades. Here, Benjamin identified the arcade as an indeterminate threshold between reality and fantasy-a dream world of non-conformity vis-à-vis the informal collection of 
artefacts and historicity that it contained. He contemplated various dualities - the old and new, the fixed and mobile, the accepted and the outcast, the past and future-to describe a bohemian world of interior spaces that operated at an urban scale.

\section{Speculation through Visualisation: Appropriations, Stratifications, Collections, and Nomadisms}

In recent years, increasingly sophisticated approaches to interior visualisation reveal an awareness similar to Walter Benjamin's preoccupation with the spatial complexity of temporal regimes. Like Benjamin, this creative work embraces the zeitgeist, but also acknowledges the value of designed objects and spatial artefacts from the past. It demonstrates greater attention to context and time, as well as to the corresponding effects that such influences can have on rendering speculative interventions. Furthermore, notable methods benefit from the compositional techniques of collage and an interest in spatial inheritance. This dialectic approach is characterised by new interventions set within existing conditions and suggests that one aspect (the new) cannot fully exist without the other (the old).

As a form of spatial thinking that operates through visual culture, dialectical interior visual representation techniques compel the designer to animate and reconcile newness with subtleness and latency. The speculative nature of this emerging work behoves design theorists to imagine a framework in which to identify, group, and critique emerging methods. Using taxonomic categories, I propose that the atmospheric spatial nuances identified by Walter Benjamin may be correlated with the post-structuralist plateaus of Deleuze and Guattari to define the parameters of a cataloguing system based on similar conditions. Translated as an ontological framework in which to lens the fundamental nature of our built environment, the plateaus provoke an awareness of emerging spatial configurations. These visualisation types engage dynamic environments-or temporal regimes-that have been shaped by context, rituals, and practices co-existing with fixed conditions.

By challenging the conventional architectural bias for hegemony and its linear relationship to time, I propose a taxonomic system that orients itself to the more nuanced practices of spatial design. As a lexicon of heterogeneous forces informed by the theories of Benjamin, Deleuze, and Guattari, I have identified four categories - appropriations, stratifications, collections, and nomadisms - to describe unique spatial relationships found within alternative forms of visualisation. 


\section{Appropriations}

The material traces of former occupancies acquire a critical dimension when they are acknowledged, assessed, curated, and incorporated into new design propositions. The act of appropriation is often evident in design projects that engage obsolescence, adaptive reuse, and interiors. As evidenced in the philosophical ponderings of Benjamin, Deleuze, and Guattari, appropriated spaces reveal the intersectional forces of production, consumption, and multiplicity of authorship in the built environment. 'Appropriative' design practices assume that cities, buildings, and interiors are hybrid productions mobilised not only by designers, but through the cultural traditions, social practices, and autonomous interventions of users. By reframing the design of the built environment in the inverse the quotidian impact of people reshaping space - appropriations celebrate marks of occupancy and ownership.
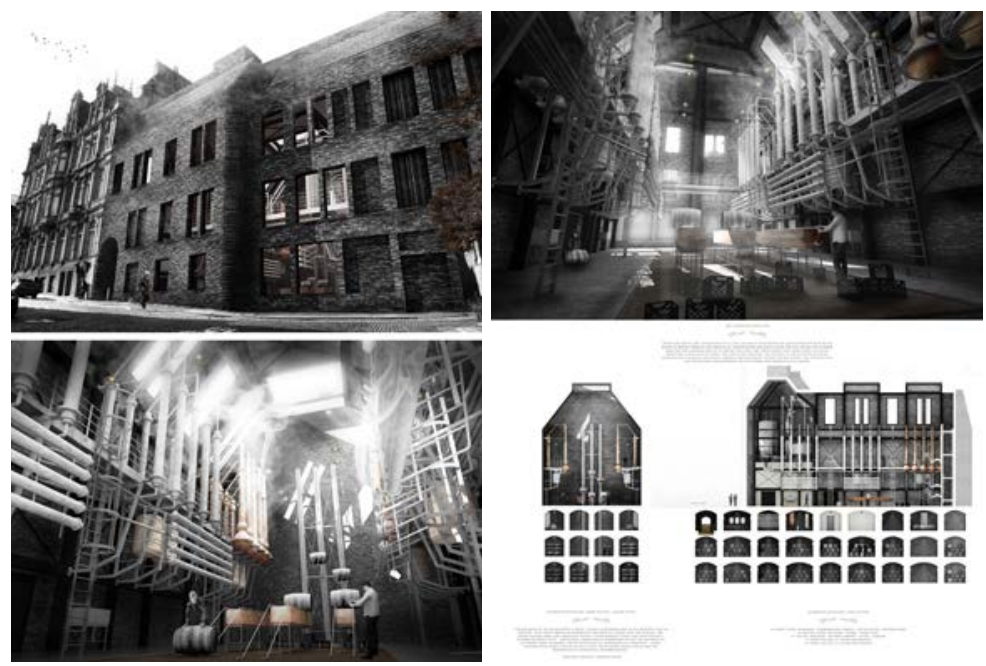

Figure $1 \mathrm{a}-1 \mathrm{~b}$ The Augmented Distillery, Matt Drury (Image courtesy of the Unbuilt Visions and Housing Tomorrow Competitions of d3 New York)

Guided by notions of acquisition and remobilisation, appropriations are highly tuned to traces and former occupancies. This visualisation type often engages highly charged imagery juxtaposing banal obsolescence alongside futurist utopianism. The content of appropriative work includes contested environments, as well as latent (historical) and curated (archival) conditions. The maker of this visualisation type recognises that meaning is inexorably embedded within the spaces and objects of the pre-existing context. As a form of ethnographic research, appropriations explore the relationship between everyday actions and new design interventions. 
Figure 2a-2b One Home for All the Homeless, Andjela Karabasevic (Image courtesy of the Unbuilt Visions and Housing Tomorrow Competitions of d3 New York)
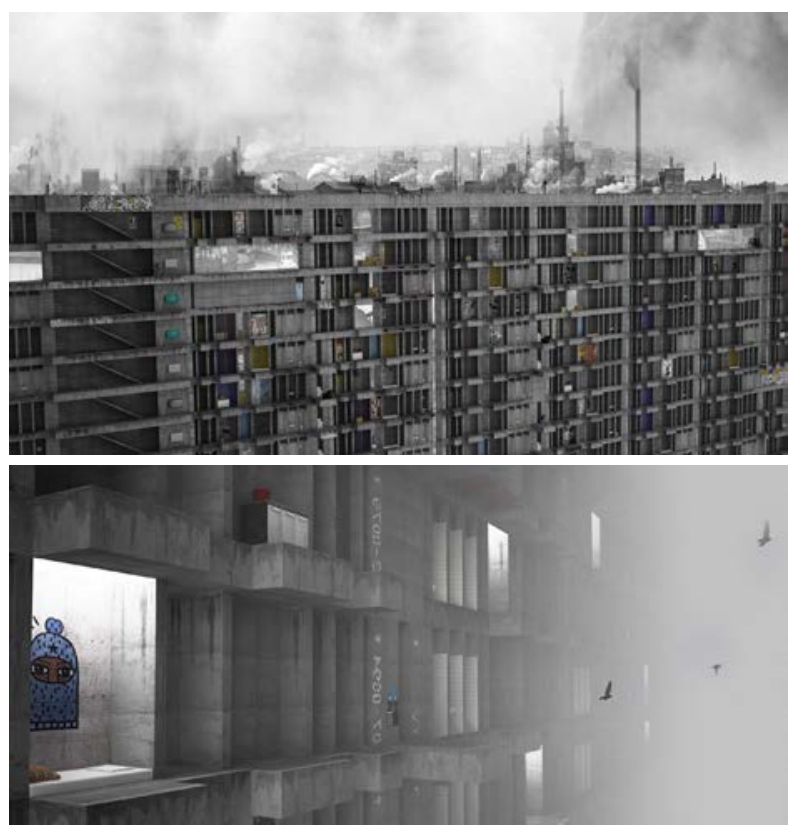

Flgure 3a-3b Infrastructure

Flows: An

Adaptation to the Brooklyn-Queens Expressway, Young Bum Kim \& Hun Kit Yuen (Image courtesy of the Unbuilt Visions and Housing

Tomorrow

Competitions of d3 New York)

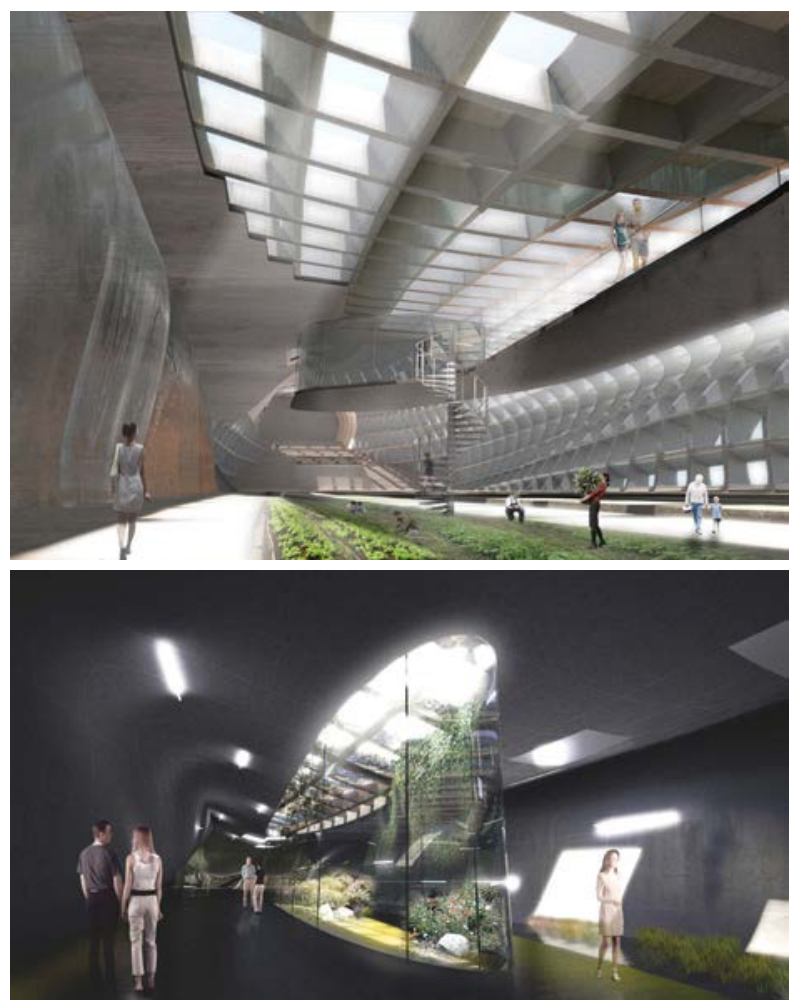




\section{Stratifications}

For Deleuze and Guattari, notions of 'strata and stratification' in the plateaus describe processes of shaping matter akin to the layering of geological formations. Stratifications are characterised by accretions and continual change. Deleuze and Guattari state:

They (strata) consist of giving form to matters, of imprisoning intensities or locking singularities into systems of resonance and redundancy, of producing upon the body of the earth molecules large and small and organising them into molar aggregates. Strata are acts of capture; they are like 'black holes' or occlusions striving to seize whatever comes within their reach. They operate by coding and territorialisation upon the earth; they proceed by code and by territoriality. The strata are judgments of God (but the Earth, or the body without organs, constantly eludes that judgment, flees and becomes destratified, decoded, deterritorialised. (Deleuze \& Guattari, 1980)

This understanding of stratification may be correlated with the spatial lexicon developed by Benjamin to describe the nineteenth-century arcades-spaces characterised by architecture, materials, occupancy patterns, and histories - that had acquired new meaning by shifting from fashionable to subversive. Like stratification, these elements are articulated through accretion into larger bodies and systems; they are connected to other systems, yet internally-focused and autonomous. Beguiling the senses, this visualisation type is often embodied by utopian/dystopian constructs of mythic proportions.
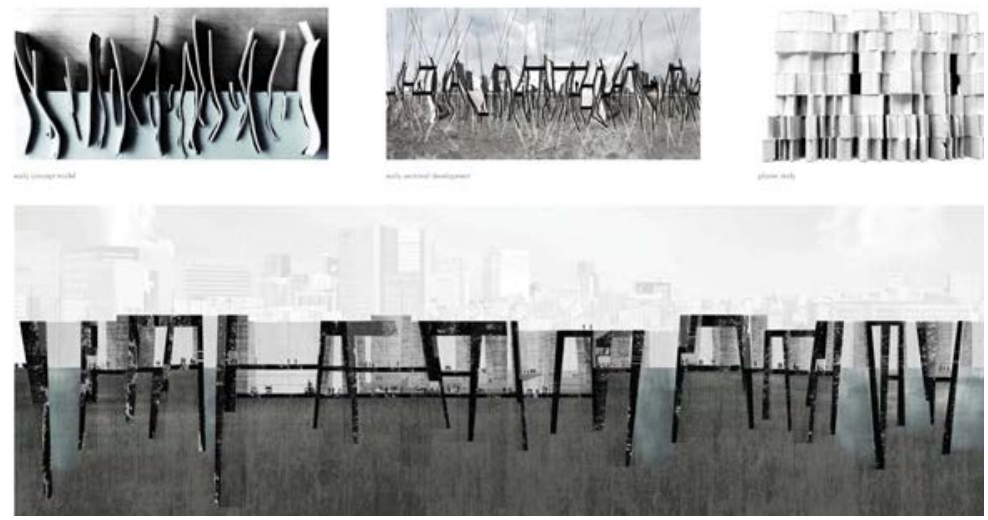

Figure $4 a$

Rooting Tsukiji, Eric Wall, Ellen Hart (Image courtesy of the Unbuilt Visions and Housing Tomorrow Competitions of d3 New York)

Strata and stratification may be brought to bear on contemporary interior visualisation practices that seek internal hegemony. Stratifications are substantially privileged toward interiority; they reject the primacy of the existing envelope by tempering its dominance. 
The content of stratifications includes exteriorless interiorsliteral excavations-as well as pure (hegemonic) and acontextual (autonomous) speculations. These visual representation practices describe internal constellations (contents) seek autonomy from larger architectural envelopes (containers).

Figure $4 \mathrm{~b}$

Rooting Tsukiji, Eric Wall, Ellen

Hart (Image

courtesy of the

Unbuilt Visions

and Housing

Tomorrow

Competitions of

d3 New York)
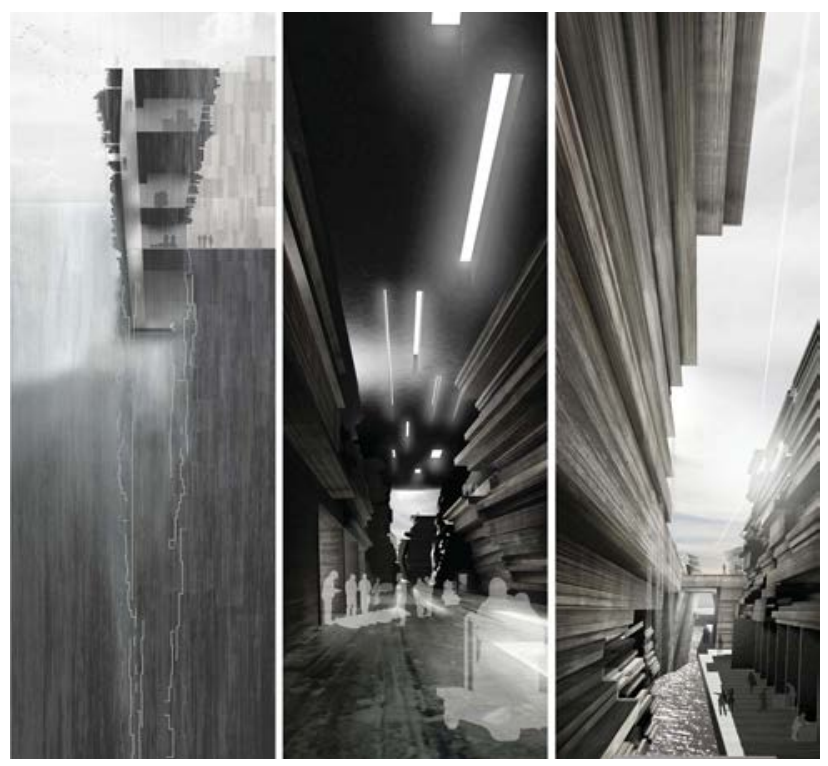

Figure $5 a-5 b$ Evacuation

Sphere: Saving Japan from

Disaster, Jeong Youngjin (Image courtesy of the Unbuilt Visions and Housing

Tomorrow

Competitions of d3 New York)
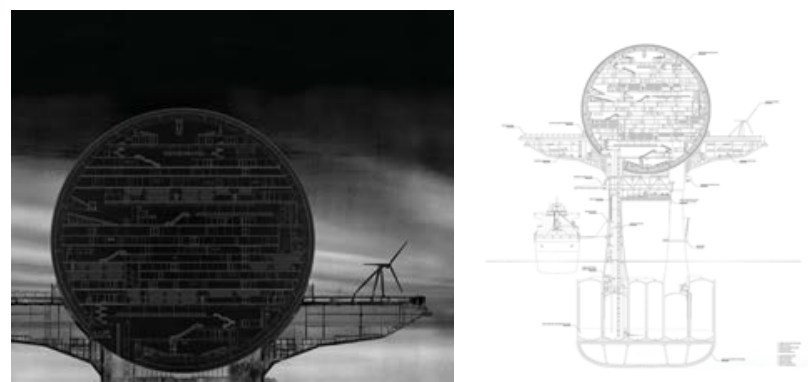

\section{Collections}

'Milieu' implies a technical term combining three meanings in French that connote surroundings, medium, and middle. Deleuze and Guattari employed the notion of the milieu and simultaneity of its meanings to critique socio-cultural processes and spatial complexity. Their analysis of these conditions is shared with Benjamin in his assessment of accretions found within the faded and obsolete arcades of interwar Paris. He states: "The first iron constructions, the first factory buildings, the earliest photos, objects 
that have begun to be extinct, grand pianos, the dresses of five years ago, fashionable restaurants when the vogue has begun to ebb from them..." (Benjamin, 1999). The atmosphere of these transitory spaces-daylit and shrouded in patina-represents a dialectical image of multiple conditions that have been archived and organically curated as an unintentional collection.

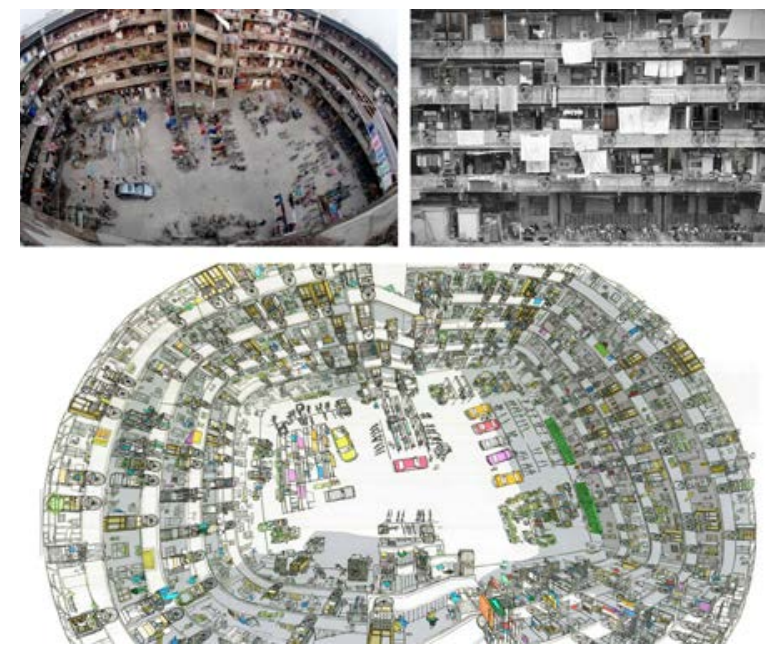

Figure 6a

Biking/Living, Liu Qicai, Yue Kang, \& Zuo Long (Image courtesy of the Unbuilt Visions and Housing Tomorrow Competitions of d3 New York)

Similarly, 'collective' interior visualisation practices demonstrate a comparative dialogue between the found and the introduced. Expressing an inherent duality in these conditions not unlike the readymade re-contextualisations of Marcel Duchamp, 'collections' negotiate a path between archival processes and speculation. Manifested in highly-charged and eclectic re-readings, this visualisation type often curates ruins-unrealised speculations or throw-away environments - to propose a layered place made new. In other words, 'collective' practices simultaneously relate to Benjamin's notion of the constellation, as well as the ontological framework of the assemblage plateau proposed by Deleuze and Guattari. Their theory of assemblage establishes an analytical approach to complexity which emphasises fluidity and multiplicity between entities and their connectivity to physical space. Furthermore, the term assemblage traces its etymology to the French word agencement, or 'arrangement,' which implies a relationship to organisation, context, and meaning. Furthermore, assemblage theory proposes that the relationship of component parts to the whole are not fixed and stable, but rather, dynamic and contingent upon connections to exteriority. Collections, therefore, describe the integration and arrangement of myriad elements that establish context and meaning. 


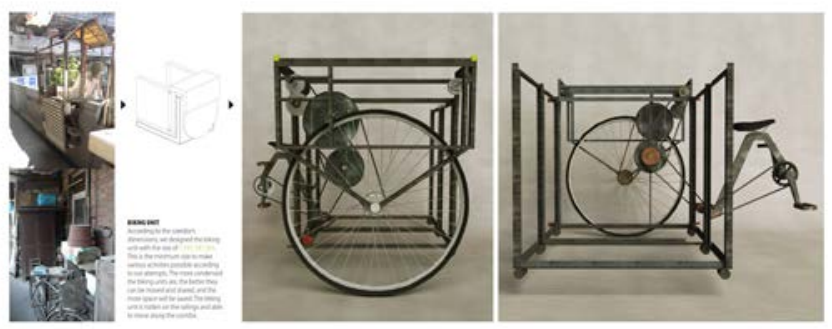

Figure $6 \mathrm{~b}$ Biking/Living,

Liu Qicai, Yue

Kang, \& Zuo

Long (Image

courtesy of the

Unbuilt Visions

and Housing

Tomorrow

Competitions of d3 New York)

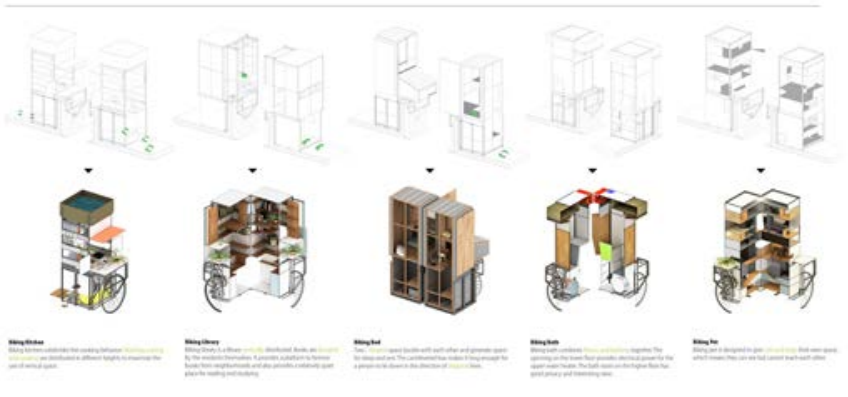

Figure $7 a-7 b-7 c$

Value Added

Landscapes,

Marc Medland

\& Steven

Rabet (Image

courtesy of the

Unbuilt Visions

and Housing

Tomorrow

Competitions of

d3 New York)
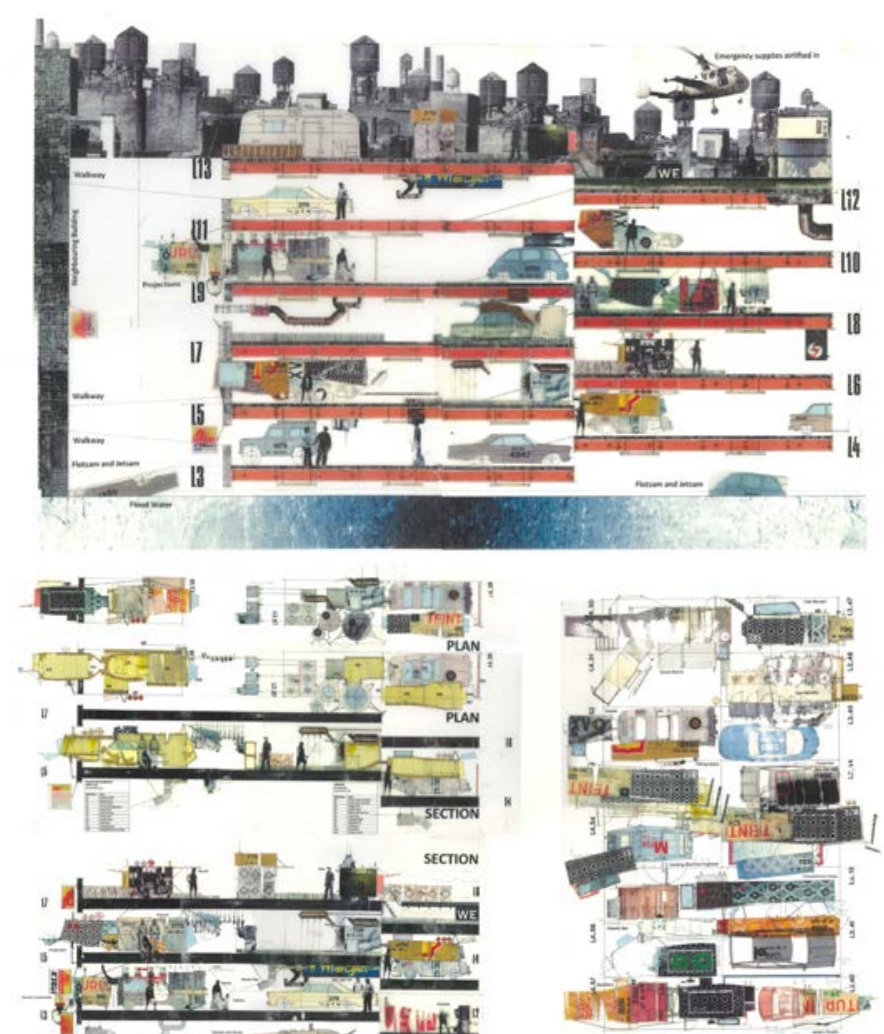
Negotiating found spaces and objects as a curatorial practice, collections are inherently anti-utopian in their resistance to hegemony. The content of collections includes inanimate objects and human bodies, as well as corporeal (physical) and incorporeal (phenomenological) conditions. As an artefact of contemporary visual culture, collective visual representation practices enhance the relationship of interior spaces to human occupancies, larger territories, and historical processes.

\section{Nomadisms}

Nomadism and alienation are shared themes in both $A$ Thousand Plateaus and The Arcades Project. For Deleuze and Guattari, nomadism represents an unstructured way of life that challenges the organisational formalities of the State. They frame the etymology of the word transient as:

The nomad has a territory; he follows customary paths; he goes from one point to another; he is not ignorant of points (water points, dwelling points, assembly points, etc.). But the question is what in nomad life is a principle and what is only a consequence. To begin with, although the points determine paths, they are strictly subordinated to the paths they determine, the reverse happens with the sedentary. The water point is reached only in order to be left behind; every point is a relay and exists only as a relay. A path is always between two points, but the in-between has taken on all the consistency and enjoys both an autonomy and a direction of its own. The life of the nomad is the intermezzo. (Deleuze \& Guattari, 1980)

Deleuze and Guattari extend the meaning of nomadism to correlate with smooth space, or environments of free movement by stating:

Smooth space is filled by events or haecceities, far more than by formed and perceived things. It is a space of affects, more than one of properties. It is haptic rather than optical perception. Whereas striated forms organise matter, in the smooth materials, signals force and serve as symptoms for them. It is an intensive rather than extensive space, one of distances, not of measures and properties. Intense Spatium instead of Extensio. A Body without Organs instead of an organism and organisation. (Deleuze \& Guattari, 1980)

Their framing of nomadism as 'molecular,',minor,',micropolitical', and 'deterritorialised' is characterised by freedom, chaos, and limitlessness. They assert that sedentary and nomadic orientations define the primary ways in which humans use space and relate to land. 
Nomads are detached from social systems, and thus, they pursue an interstitial existence that may be collective, singular or a hybrid of both conditions. Nomads actively critique stasis by pursuing autonomy and change; their relationship to space is highly temporal. For Benjamin, nomadism defined his life as a philosopher-critic who studied transience and the role of the present in historical remembrance. He defined an alternative relationship between migration to territories - of people moving between points of dwelling, production, assembly, and leisure. Similarly, this way of living and engaging with the built environment may be applied to 'nomadic' visualisation practices. The nomadic taxonomy examines transience and its impact on fixed spaces; nomadisms are inherently open and dynamic. The content of nomadic work includes human bodies and dynamic environments, as well as corporeal (physical) and incorporeal (phenomenological) conditions. Much like Archigram's Walking City, nomadic visualisations often depict deployable environments and how the interiority of such constructs would be perceived from beyond. As a visual representation medium embracing disruptive forces, nomadic practices illustrate the relationship of interiors to motion and temporality.

Figure $8 \mathrm{a}-8 \mathrm{~b}$ Wave Cube: Turkish Pavilion for Expo Yeosu, Onat Oktem, Zeynep

Oktem, \& Ziya Imren (Image courtesy of the Unbuilt Visions and Housing Tomorrow Competitions of d3 New York)
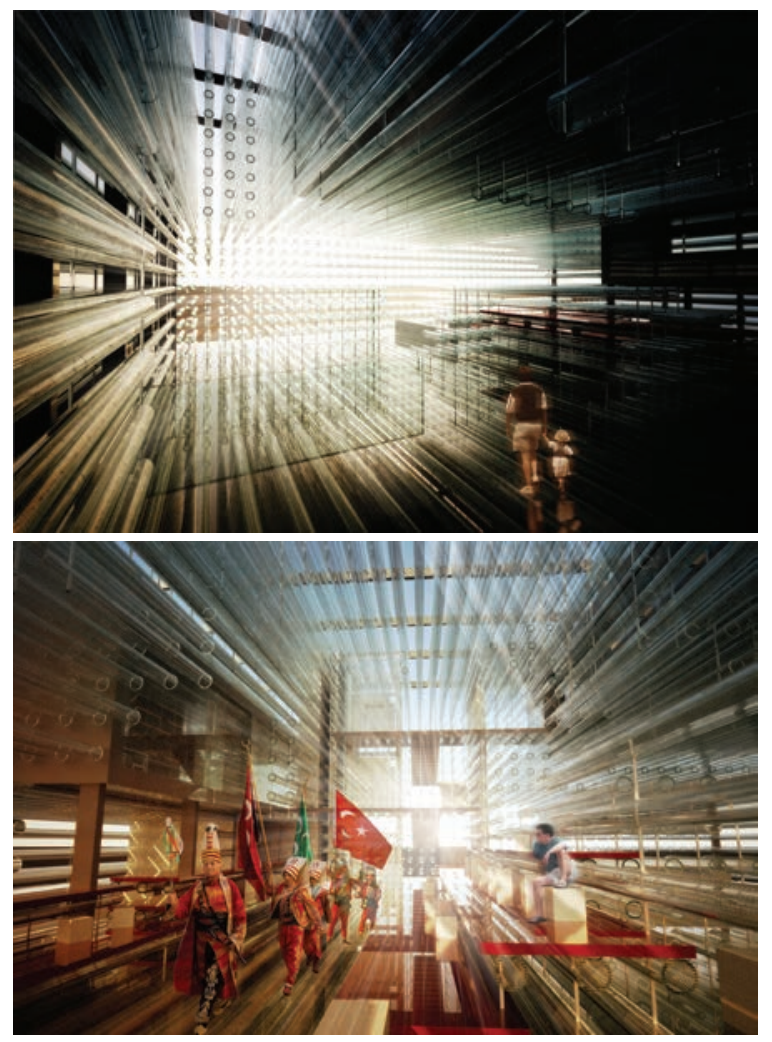

Gregory Marinic 

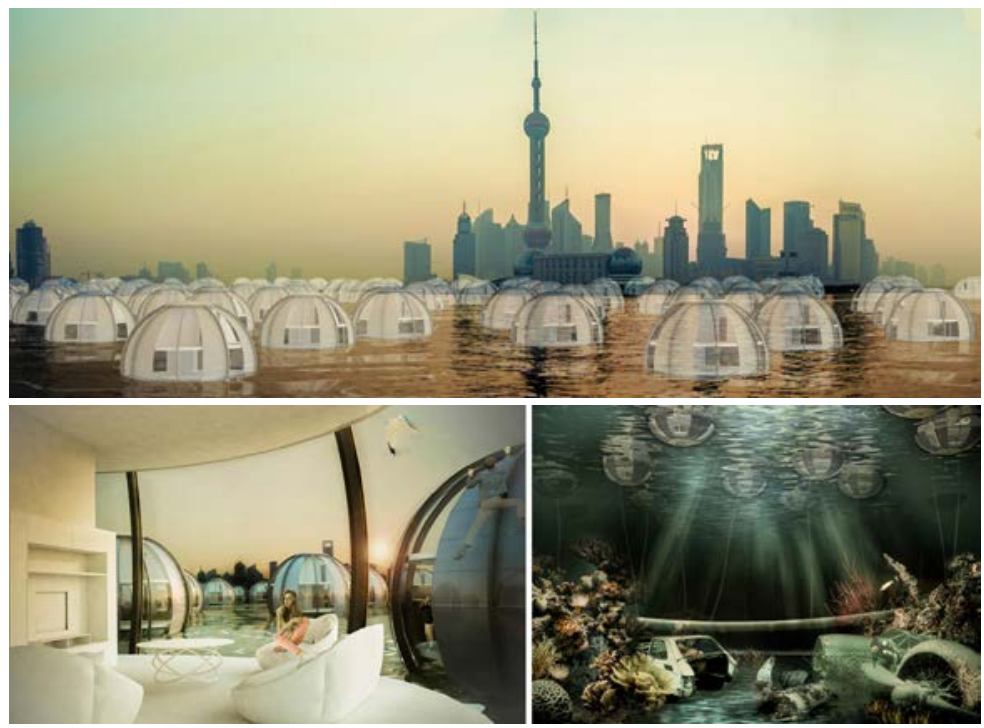

\section{Conclusion}

Contemporary visual representation practices are increasingly redefining the role of representation in design speculation. As such, the temporal nature of buildings and socioeconomic change fuels an ongoing reassessment of how obsolescence and regeneration impact interior spaces. Evidenced by the preceding exemplars, design interventions leveraged within spatial inheritance often shape a more critical approach to reuse and interiority.

Inspired by theorists Benjamin, Deleuze, and Guattari, I propose the taxonomic lexicon presented here to confront conventional interior visualisation practices. It can provoke more critical forms of inquiry by asking practitioners, academics, and students to acknowledge the contextual conditions and informal marks that the built environment acquires over time. The examples included herein reveal the agency of people and their imprint on buildings; this body of creative work compels architects and designers to rethink their agency. Makers should work with a simultaneous lens on the past and toward the future by assuming the inevitability of change, flexibility, and responsiveness. Greater empathy for spatial inheritance inspires a more nuanced approach to design through actions that acknowledge latent conditions through stewardship and advocacy.

The next generation of architects and designers must be prepared to promote more contextually diverse and adaptable approaches
Figure $9 a-9 b$ Sea Deployable: Bubble Breath I, M. Borowik, M. Czarny, A. Rakowska, \& K. Sosinska (Image courtesy of the Unbuilt Visions and Housing Tomorrow Competitions of d3 New York) 
in the built environment. As such, it is important for architects and designers to understand the pre-existing contextual narratives of their projects and how these spaces may be more provocatively catalysed through visualisation. In this regard, specialised visualisation practices are advancing more contextually engaged conceptual design processes. Most importantly, critical forms of interior visualisation-introduced during the foundation level to students of architecture, interiors, urban design, historic preservation, and adaptive reuse-provide opportunities for young designers to contemplate their stewardship of spatial inheritances and society at an interior scale.

\section{References}

Benjamin, W. (1999). The Arcades Project: Walter Benjamin (H. Eiland \& K. McLaughlin, Trans.). Cambridge: Belnap Press of Harvard University Press. (Original work published 1982)

Chisholm, D. (2005). Queer constellations: Subcultural space in the wake of the city. Minneapolis: University of Minnesota Press.

Deleuze, G. \& Guattari, F. (1980). A thousand plateaus: Capitalism and schizophrenia. Paris: Les Éditions de Minuit.

Horton, J. \& Kraftl, P. (2014). Cultural geography: An introduction. London: Routledge.

Lunn, E. (1984). Marxism and modernism: An historical study of Lukács, Brecht, Benjamin, and Adorno. Oakland: University of California Press.

Ross, A. (2016). Walter Benjamin's concept of the image. London: Routledge.

Shields, R. (1994). Fancy footwork: Walter Benjamin's notes on flânerie. In K. Tester (Ed.), The flâneur (pp. 61-80). London: Routledge. 